\title{
Fuzzy Control and Simulation of Vibration Isolation of Engine with
}

\section{Magneto Rheological Mounts}

\author{
Xiao-e Wang ${ }^{1, a}$ \\ ${ }^{1}$ Department of Electromechanical Engineering, Leshan Vocational and Technical College, Leshan, \\ Sichuan, China, 614000 \\ a email
}

Keywords: Magneto rheological Mount, Fuzzy Control and Simulation, Vibration Isolation

\begin{abstract}
With the development of science and technology, coupled with the continuous lowering of the mass of the car itself, the problems of noise、 vibration、 harshness ( $\mathrm{NVH}$ ) caused by engine vibration attracted more people's attention. This paper firstly clarifies the concept and principle of magneto rheological mounts and then explores the fuzzy control and simulation of vibration isolation of magneto rheological mounts in order to provide references for the related researchers.
\end{abstract}

\section{Introduction}

At present, the engine is generally used for passive rubber mount vibration isolation. In the low frequency section, it has a better effect. But at high frequency, the dynamic stiffness can increase the dynamic stiffness, which cannot meet the requirements of high frequency vibration isolation and noise reduction. In recent years, the study of hydraulic mount, there may be low frequency damping, high frequency dynamic liquid and so on. Controllable fluid suspension is an important development direction of the current power isolation device, commonly used electric and magnetic fluid as a damping control vector, but only the isolator can effectively adjust the controllable damping, stiffness, vibration isolation effect even if the establishment of control model perfect acquisition is limited. In recent years, the magneto rheological elastomer, which has the advantage of wide band and controllable magnetic field, can be controlled by the external magnetic field. Therefore, with MR damper and parallel variable stiffness of magneto rheological elastomer and variable damping of engine mounting, the vibration isolation system stiffness and damping control at the same time, is expected to allow the engine to achieve effective isolation in a wide frequency range. Under different engine speed, the stiffness and damping of the suspension system can change the frequency ratio and the damping ratio of the vibration isolation system, so as to change the energy transfer from the engine to the vehicle body. The relationship between suspension stiffness, damping and vibration characteristics of the complex system, cannot establish accurate mathematical model, it is necessary in the broadband excitation, using fuzzy control method in a controller with adjustable parameters, to explore the use of magneto rheological damping and stiffness adjustable suspension of engine vibration isolation..

\section{Concept and Principle of Magneto Rheological Mounts}

Concept. Magneto rheological fluid is a kind of controllable fluid under magnetic field can achieve a substantial increase in viscosity, and showed the yield phenomenon of solid, MRF is continuous and reversible regulation, reflect the time in milliseconds, easy to control, widely used in hydraulic suspension using liquid inertia in the flow passage the increase of engine suspension 
damping, the magneto rheological fluid contained in the traditional hydraulic suspension, with controllable viscosity characteristics of MR fluid, increase the suspension damping, the damping of the domestic application of magneto rheological fluid in the car on the main magneto rheological fluid damper and magneto rheological fluid suspension, for the study of magnetic mount are the main flow mode, shear mode and squeeze mode only with controllable channel based on engine mounting. This paper attempts to study a new type of magnetic rheological fluid with controllable channel and the inertia track mount. The inertia channel of magneto rheological fluid is controlled by magnetic field. The purpose is to further increase the low frequency damping suspension, based on the new structure of magneto rheological suspension. Drawn from the theoretical analysis and test and analysis of the suspension dynamic stiffness and damping curve, and establish the control model to predict the damping effect of magneto rheological fluid suspension.

Principle. There are three kinds of working modes, which are flow mode, shear mode and extrusion mode. As shown in Figure 1, the liquid flow in the channel is laminar, and the effect of the side wall of the channel is ignored. We can get the relationship between the pressure drop and the current and the liquid resistance formula of the controllable channel.

$$
\begin{aligned}
& \Delta P_{\mathrm{m}}=\frac{24 \eta_{0} l}{w h^{3}}+\frac{6 l}{h} \alpha\left(\frac{N I}{2 h}\right)^{\beta} \\
& R_{m}=\frac{\Delta P_{\mathrm{m}}}{\Delta Q_{\mathrm{m}}}=\frac{24 \eta_{0} l}{w h^{3}}+\frac{6 l}{Q_{m} h} \alpha\left(\frac{N I}{2 h}\right)^{\beta}
\end{aligned}
$$

Among them, $\eta_{0}$ is the magneto rheological fluid zero field viscosity; $\Delta P_{\mathrm{m}}$ is the controllable channel pressure drop; $l$ is the controllable channel length; $w$ is the controllable channel for perimeter ring; $N$ is the number of turns of the coil; $I$ is the intensity of the current controlled channel; $Q_{m}$ is the liquid flow; $h$ is the controllable channel clearance.

\section{Fuzzy Control of Vibration Isolation of Magneto Rheological Mounts}

In the case of a certain quality of the engine, if the adjustment of suspension stiffness, suspension will change the natural frequency, critical damping coefficient, and then change the vibration isolation system of frequency ratio and damping ratio; if the adjustment of suspension damping, vibration will change the damping ratio of the system; and the excitation frequency change, will change the vibration system frequency ratio. Thus, at different engine excitation frequency, stiffness and damping control of vibration isolation system, it will change the frequency ratio and damping ratio, but the relationship between these variables is complex, cannot establish accurate mathematical model, the control method on the model, it is difficult to realize isolation in broadband excitation of the engine. Fuzzy control does not need accurate model, which is expected to solve the vibration isolation control problem with variable stiffness and damping. But because the engine vibration isolation system has a certain dynamic uncertainty in broadband excitation in various suspension under dynamic load, the suspension stiffness and damping variable and is not the same, the model parameters are time-varying and nonlinear characteristics, which requires the adaptive fuzzy controller. On line, the quantitative factor and scale factor can be adjusted to change the output characteristic of the fuzzy control system, and it is an effective method to realize the adaptive fuzzy control. When the vibration velocity of a point of engine mounting negative maximum (vertical positive), and the acceleration is negative to the maximum, the fastest speed of 
the vibration attenuation is zero, it should make the damping value reached the maximum value, the maximum damping force; similarly, when a point of engine mounting vibration the maximum speed, acceleration and maximum damping, also should take the maximum, resulting in a negative maximum damping force. The fuzzy control rule of damping forces is shown in Table 1.

Table 1.Fuzzy control rule of damping forces

\begin{tabular}{|l|l|l|l|l|l|l|l|}
\hline & NB & NM & NS & ZE & PS & PM & PB \\
\hline NB & PB & PB & PB & PB & PM & PS & PS \\
\hline NM & PB & PB & PM & PM & PS & PS & ZE \\
\hline NS & PM & PM & PS & PN & ZE & ZE & ZE \\
\hline ZE & PS & PS & PS & ZE & ZE & ZE & ZE \\
\hline PS & ZE & ZE & ZE & NS & NS & NM & NM \\
\hline PM & ZE & NS & NS & NM & NM & NB & NB \\
\hline PB & NS & NS & NM & NB & NB & NB & NB \\
\hline
\end{tabular}

\section{Simulation of Vibration Isolation of Magneto Rheological Mounts}

Influence of Current Intensity. Under the condition that the other mounting structure size are unchanged, the variation of the input current intensity can be seen in the diagrams of Mount dynamic stiffness in the applied current rapidly increased to about, reached a peak at around $15 \mathrm{~Hz}$, and with the increase of the peak strength of the current increases. When the vibration frequency is $20 \mathrm{~Hz}$, the dynamic stiffness is increased from $350 \mathrm{~N} / \mathrm{mm}$ to $510 \mathrm{~N} / \mathrm{mm}$ by adding current and zero current, which has increased by nearly $45 \%$. It can be seen that the magneto rheological hydraulic suspension can be obtained by adjusting the size of the ideal input current in low frequency and large amplitude under the condition of dynamic stiffness, engine vibration isolation requirements of low rigidity trend. The variation of damping lag angle also shows similar trend. The curves of dynamic stiffness and damping lag angle with the change of the current intensity are shown in Figure 1.
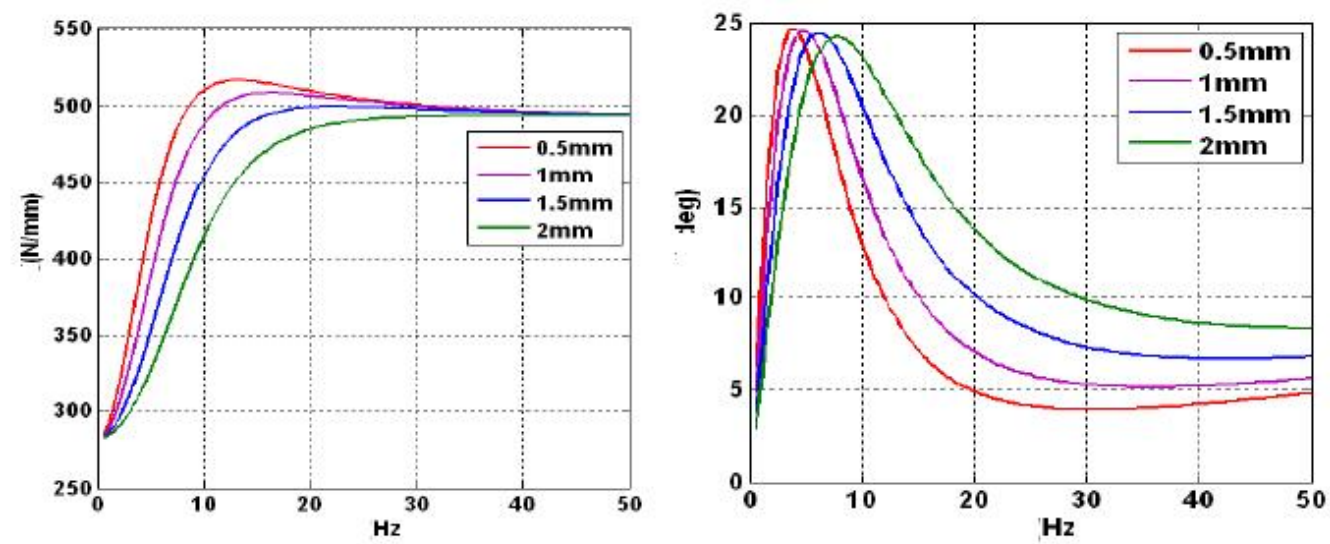

Figure 1.Curves of dynamic stiffness and damping lag angle with the change of current intensity

Influence of Channel Clearance. In the case of the same input current and other structures, the peak value of the suspension stiffness decreases with the increase of the controllable channel gap, and the peak frequency shifts to the right, reaching a stable value at $30 \mathrm{~Hz}$. The peak size decreased from $520 \mathrm{~N} / \mathrm{mm}$ to $480 \mathrm{~N} / \mathrm{mm}$, which decreased by nearly $8 \%$. The damping angle peak frequency shifts to the right with the increase of the clearance, and the peak value decreases by about $4.2 \%$. Taking into account the magneto rheological fluid viscous, controlled channel gap cannot be too 
small, otherwise it will affect the flow of the liquid, also lost the hydraulic mount meaning, to meet the engine vibration isolation requirements, select the appropriate clearance. Its value is generally $1 \mathrm{~mm}-2 \mathrm{~mm}$. The curves of dynamic stiffness and damping lag angle with the change of the channel clearance are shown in Figure 2.
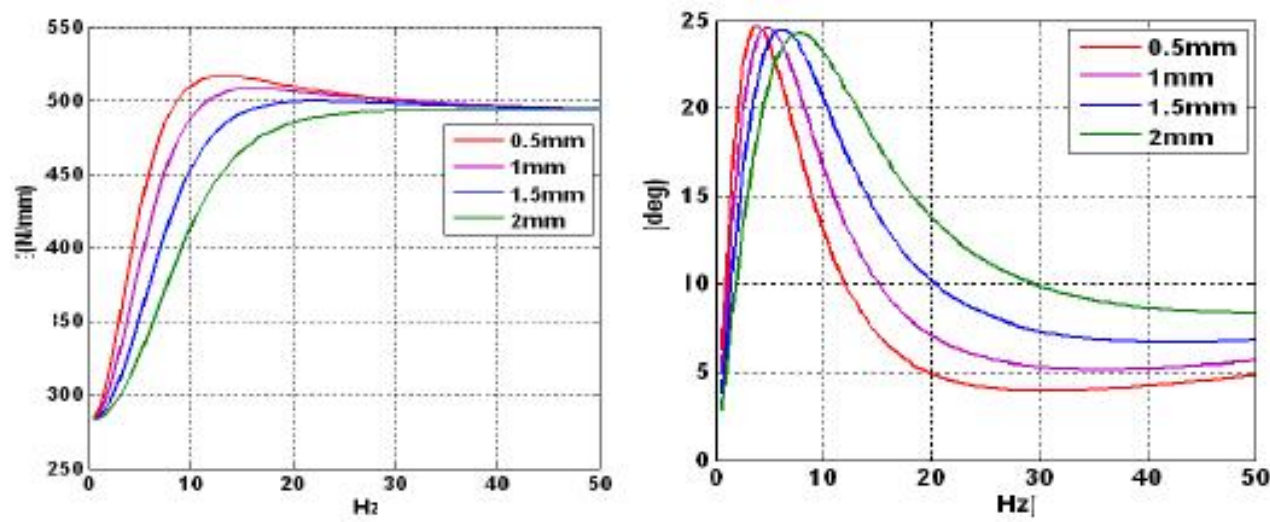

Figure 2.Curves of dynamic stiffness and damping lag angle with the change of channel clearance

Influence of Cross-Sectional Area of Inertia Channel. In the case of the same input current and other structures, it can be seen that the suspension stiffness increases with the increase of the cross section area of the inertia channel, the peak frequency shifts to the right, and the peak value increases obviously. Area under the $510 / \mathrm{mm} \mathrm{N}$ increased to $580 / \mathrm{mm} \mathrm{N}$, an increase of nearly $14 \%$. It can be seen that the increase of cross section area is equivalent to reducing the liquid sense of the inertia channel, so that the resonance frequency is increased, that is, the reason why the peak frequency moves to the right. The damping lag angle also increases with the increase of the cross section area of the inertia channel, and the peak frequency shifts to the right. The damping angle increased from 25 degrees to degrees, which increased by nearly $37.5 \%$. The reason is the increase in cross-sectional area and the flow of liquid between the upper and lower liquid chamber increases. The curves of dynamic stiffness and damping lag angle with the change of the cross-sectional area of inertia channel are shown in Figure 3.
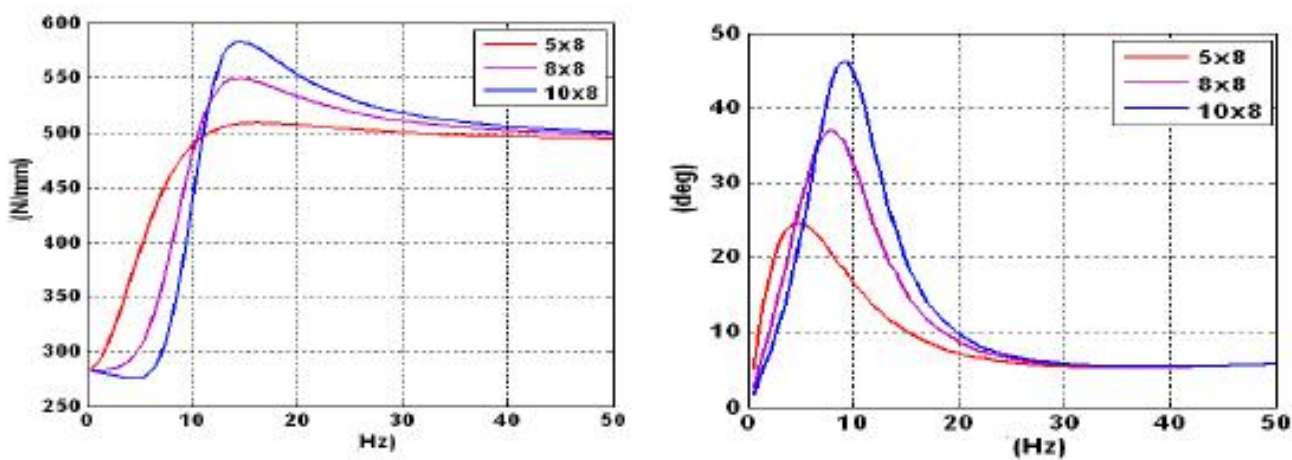

Figure 3.Curves of dynamic stiffness and damping lag angle with the change of cross-sectional area of inertia channel

\section{Conclusion}

Magneto rheological fluid is a kind of intelligent material with the change of its rheological properties under the action of external magnetic field. This paper carries on the simulation of the engine magneto rheological vibration system. The simulation results show that under the condition 
of the low frequency, the suspension dynamic stiffness and damping increase with the increasing input current. We can improve the suspension structure and the high frequency dynamic characteristics of the suspension to perfect the magneto rheological suspension.

\section{Acknowledgements}

This research is the results of the "Scientific Research Project in Sichuan Province Department of Education”. The Project Number is 15ZB0400.

\section{References}

[1] Shi Wenku, Hou Suojun, Wang Xuejing, Wang Shaohua, Li Haisheng, Transactions of the Chinese Society of Agricultural Engineering, Vol. 28(2012) No 20, p.50-57

[2] Li Rui, Chen Weimin, Liao Changrong, Liu Huibing, Li Yinguo, Journal of System Simulation, Vol. 21(2009) No 4, p.944-953

[3] Liu Huibing, Liao Changrong, Li Rui, Luo Jing, Li Xing, Jian Xiaochun, Journal of Vibration, Measurement \& Diagnosis, Vol. 31(2011) No 2, p.180-184

[4] Zhang Ziwei, Zheng Ling, Deng Zhaoxue, Fu Jianghua, Guo Minmin, Automotive Engineering, Vol. 36(2014) No 10, p.1267-1273 